\title{
vibrations engendrées par le compactage dynamique
}

par

P. Liausu

Techniques Louis Ménard S. A.

RESUME -

La méthode d'amélioration des sols par compactage dynamique repose sur la transmission de chocs de forte energie au terrain, chocs qui vont aussi engendrer des vibrations aux ouvrages éventuellement avoisinants. Par analyse de mesures effectuées sur un certain nombre de chantiers, cet article met en évidence des lois d'atténuation de ces vibrations, permettant ainsi d'estimer à l'avance le niveau de sollicitation des ouvrages situés à proximité de la zone à compacter.

\section{INTRODUCTION}

Depuis plus d'une dizaine d'années maintenant, la consolidation dynamique est de plus èn plus fréquemment employée comme méthode de densification des sols. Son principe de base consiste à transmettre des chocs de forte energie à la surface d'un sol initialement compressible, afin d'en améliorer en profondeur les caractéristiques mécaniques. En pratique, il s'agit de laisser tomber en chute libre d'une hauteur de l'ordre de 15 à 20 mètres (voire 30 mètres) une masse pesant de 10 à 30 tonnes, selon un programme bien défini en fonction du site à traiter et de l'ouvrage futur. Cette opération va donc engendrer des vibrations dans les ouvrages éventuels situés à proximité du terrain à compacter.

Le présent article fait la synthèse et l'analyse de mesures réalisées sur une dizaine de chantiers executés en Rép.Féd.d'Allemagne où l'existence de normes bien précises quant aux vibrations admissibles a conduit à effectuer systématiquement une campagne de mesures lors du démarrage des travaux.

\section{RAPPEL SUR LES VIBRATIONS - NORMES}

La chute du pilon à la surface du sol engendre l'apparition de plusieurs trains d'ondes.

De la nature du sol et en particulier de la disposition des différentes couches dépendent la vitesse de propagation, l'amortissement, l'importance des ondes réfléchies, etc ... si bien que chaque chantier représente un cas particulier. Cependant, le schéma établi pour un massif élastique avec une vibration sinusoídale entretenue nous fournit une représentation acceptable du phénomène; ainsi que le montre la figure $n^{\circ} 1$, on peut distinguer trois types fondamentaux d'ondes

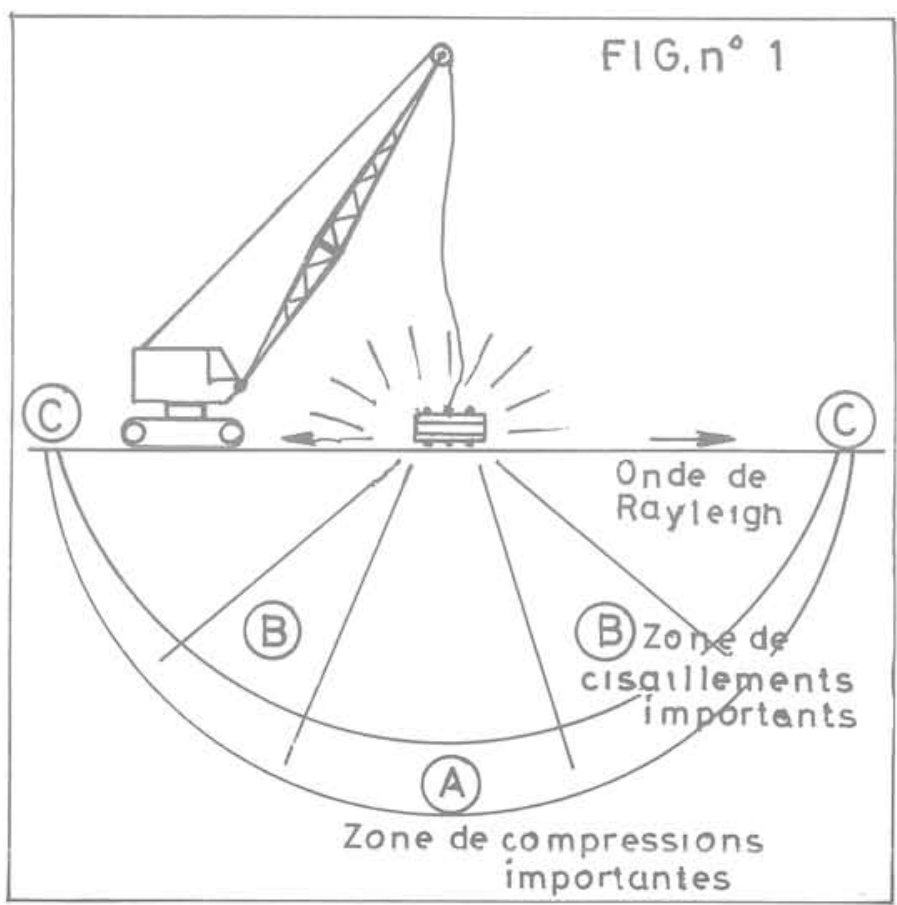

Fig. 1 Ondes engendrées par le choc 
- l'onde de compression, dont la vitesse de propagation est la plus élevée et dont l'influence se fait sentir plus en profondeur qu'en surface (zone A)

- l'onde de cisaillement, la plus importante en ce qui concerne l'effet du compactage s'exerce surtout dans la zone B ("shear window")

- l'onde de Rayleigh, une onde d'interface sol-air (zone C), est inintéressante pour le compactage proprement dit, car elle ne va pas en profondeur, mais c'est celle qui va solliciter les ouvrages avoisinants. C'est la décroissance de cette onde qui sera étudiée dans les paragraphes suivants.

Trois paramètres permettent de caractériser une vibration :

- le déplacement en fonction du temps,

- la vitesse de vibration (ou vitesse particulaire),

- l'accélération du mouvement.

Dans le cas d'un mouvement périodique sinusoidal, pour une fréquence donnée, la mesure de l'un des paramètres suffit, les 2 autres se déduisant par dérivation ou intégration très simple.

Les normes allemandes DIN 4150 (Sept. 75) fixent la vitesse particulaire résultante maximale admissible pour différents types de construction :

\begin{tabular}{|c|c|c|}
\hline classe & \multicolumn{1}{|c|}{ ouvrage } & Vr max. \\
\hline 1 & $\begin{array}{l}\text { Bâtiments industriels ou à } \\
\text { usage d'habitation en bon } \\
\text { etat }\end{array}$ & $8 \mathrm{~mm} / \mathrm{s}$ \\
\hline 2 & $\begin{array}{l}\text { Bâtiments particulièrement } \\
\text { rigides en bon etat }\end{array}$ & $30 \mathrm{~mm} / \mathrm{s}$ \\
\hline 3 & $\begin{array}{l}\text { Bâtiments qui ne tombent } \\
\text { pas dans les classes 1 et } \\
2 \text { ou particulièrement sen- } \\
\text { sibles. }\end{array}$ \\
\hline
\end{tabular}

avec $\quad \mathrm{Vr}=\left(\mathrm{Vv}^{2}+\mathrm{Vh}_{1}{ }^{2}+\mathrm{Vh}_{2}{ }^{2}\right) 0.5$

Il existe bien sûr d'autres normes ou recommandations de présentations différentes, mais toutes convergent sur le fait que des vibrations induisant des vitesses particulaires inférieures à 8 à $10 \mathrm{~mm} / \mathrm{s}$ ne doivent pas causer de dommages aux bâtiments normaux concernés.
Il convient encore de remarquer que ces normes s'appliquent à des vibrations continues; le pilonnage engendre lui des vibrations répétées mais non continues, ce qui devrait permettre de relever sensiblement le seuil de tolérance.

\section{DEROULEMENT DES MESURES}

Appareillage - Toutes les mesures citées dans cet article ont été effectuées par des organismes spécialisés indépendants, (cf. références à la fin du texte) à l'aide de chaînes de mesures constituées de géophones ou accéléromètres capables d'enregistrer des vibrations dans la plage $1-80 \mathrm{~Hz}$, d'un amplificateur et d'un dispositif d'enregistrement sur bandes magnétiques.

\section{Caractéristiques des chantiers}

\begin{tabular}{|c|c|c|}
\hline chantier & $\begin{array}{l}\text { ouvrage } \\
\text { vérifié }\end{array}$ & sol \\
\hline Breitscheid & pipeline & $\begin{array}{l}1,50 \mathrm{~m} \text { remblai, } \\
\text { sous-sol limoneux } \\
\text { mou }\end{array}$ \\
\hline Grassau & & $\begin{array}{l}2,50 \mathrm{~m} \text { gravier } \\
3,50 \mathrm{~m} \text { limon + } \\
\text { tourbe, sous-sol } \\
\text { gravier compact }\end{array}$ \\
\hline Plochingen & & $\begin{array}{c}7 \text { m remblai ordure } \\
\text { sous-sol marneux } \\
\text { dur }\end{array}$ \\
\hline Hanovre & $\begin{array}{c}\text { maisons } \\
\text { d'habita- } \\
\text { tion }\end{array}$ & $\begin{array}{l}1,50 \text { m remblai } \\
5,50 \text { m limon, } \\
\text { sous-sol gravier } \\
\text { compact }\end{array}$ \\
\hline Cuxhaven & $\begin{array}{l}\text { pylone } \\
\text { haute } \\
\text { tension }\end{array}$ & $\begin{array}{c}\text { remblai, sous-sol } \\
\text { limon+tourte très } \\
\text { mou }\end{array}$ \\
\hline Forchheim & voie ferrée & $\begin{array}{l}6 \text { m remblai ordure } \\
\text { sous-sol dur }\end{array}$ \\
\hline Reitmehring & $\begin{array}{l}\text { maisons } \\
\text { d'habita- } \\
\text { tion }\end{array}$ & $\begin{array}{c}\text { alluvions molles } \\
\text { avec qqes. bancs } \\
\text { de gravier. }\end{array}$ \\
\hline Mainz & $\begin{array}{l}\text { maisons } \\
\text { d'habita- } \\
\text { tion. }\end{array}$ & $\begin{array}{l}6 \text { - } 8 \text { m remblai } \\
\text { sous- sol limon } \\
\text { compact }\end{array}$ \\
\hline Landshut & $\begin{array}{l}\text { maisons } \\
\text { d'habita- } \\
\text { tion } \\
\text { entrepots }\end{array}$ & $\begin{array}{l}8 \mathrm{~m} \text { gravier } \\
\text { sous-sol compact }\end{array}$ \\
\hline
\end{tabular}


RESULTATS

Vitesse particulaire Vr résultante en fonc-

tion de la distance au point d'impact $d$

Tous les résultats obtenus pour des ener-

gies comparables de 200 à $300 \mathrm{t} x \mathrm{~m}$ (poids

$x$ hauteur de chute) sont regroupés sur la

figure $n^{\circ} 2$.

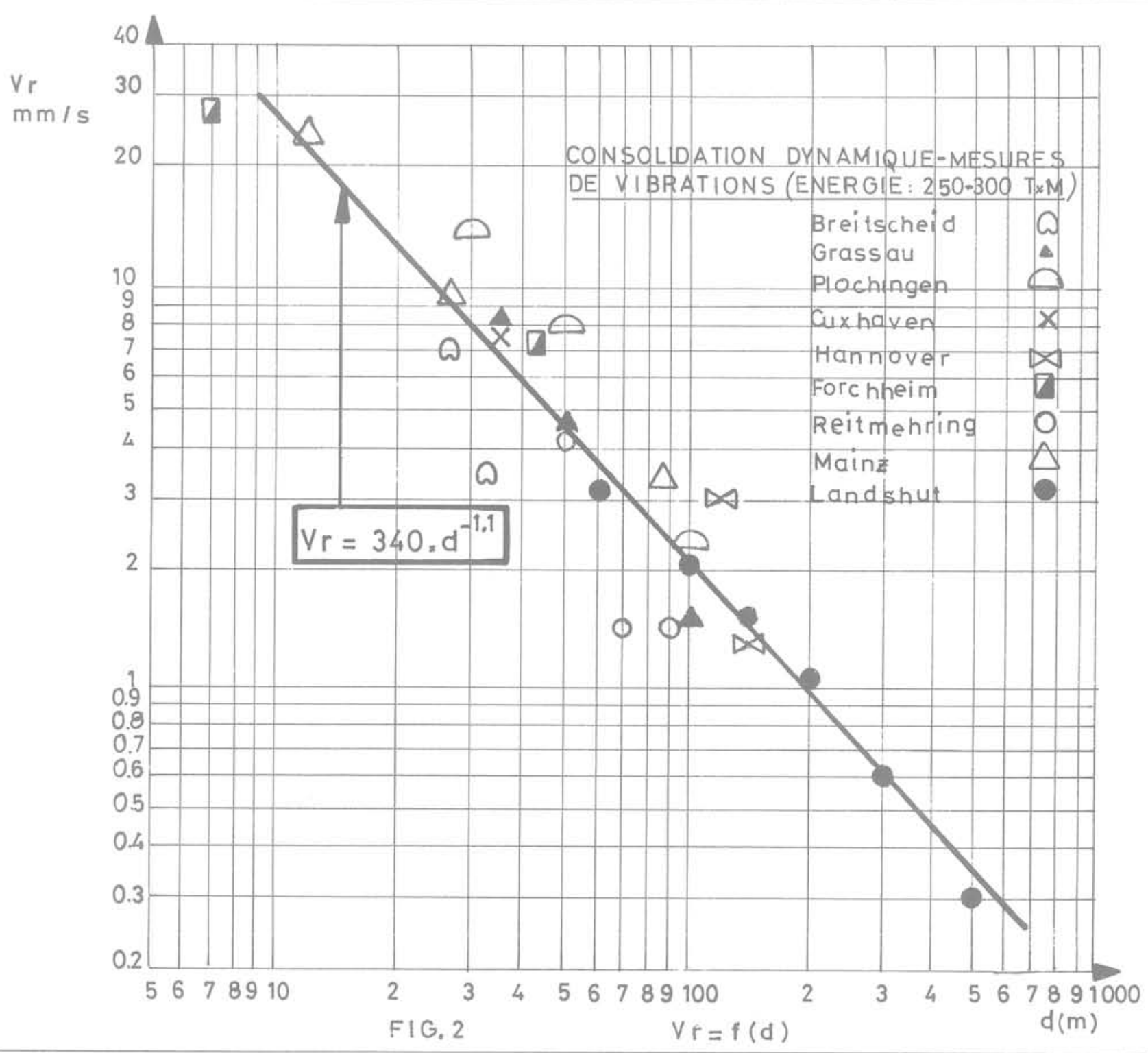

Fig. 2 Atténuation des vibrations en fonction de la distance 
On peut constater un très bon a lignement des points en coordonnées logarithmiques ce qui conduit à l'équation suivante obtenue par régression linéaire :

$$
v_{r}=340 \times \mathrm{d}^{-1 \cdot 1}
$$

( $V_{r}$ en $\mathrm{mm} / \mathrm{s} ; \mathrm{d}$ en mètres ) Le coefficient de détermination $r^{2}$ étant égal à 0,89 .

Vitesse particulaire V $r$ résultante en fonction de l'énergie unitaire

Nous présentons quelques exemples de courbes sur la figure $n^{\circ} 3$ :

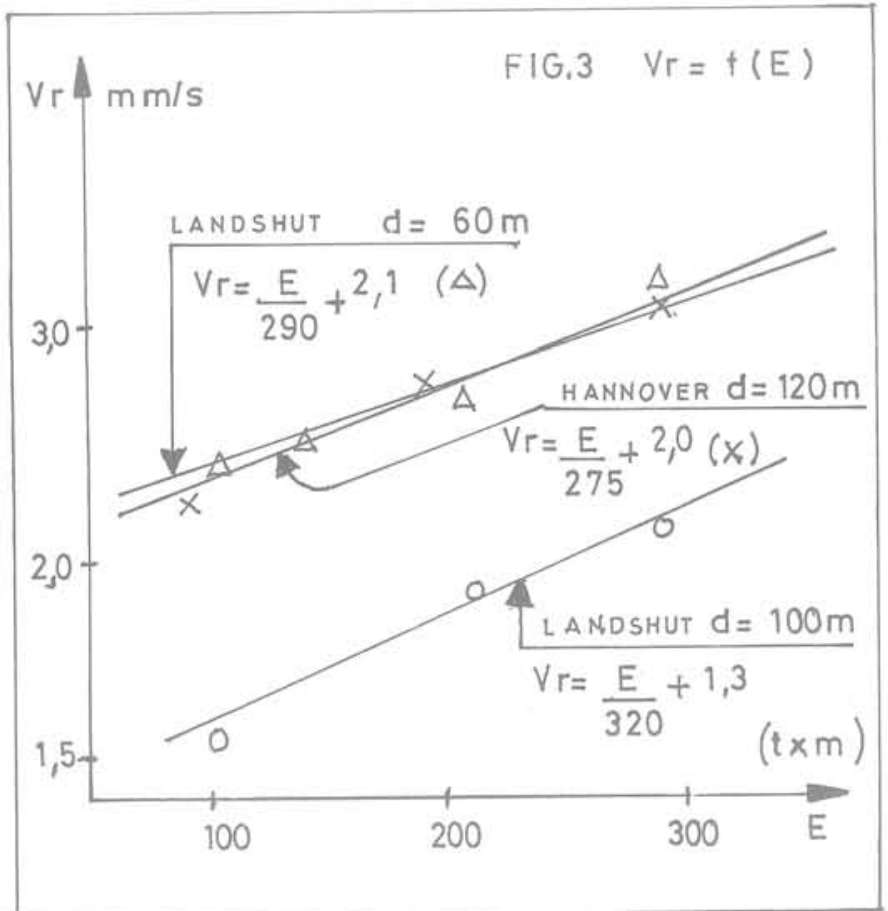

Fig. 3 - Atténuation des vibrations en fonction de l'énergie

Il est intéressant de noter que les courbes prennent l'allure de droites dont les pentes sont voisines

$$
\text { V r }=\frac{E}{A}+B
$$

$\mathrm{E}=$ énergie en $\mathrm{t} \times \mathrm{m}$

$\mathrm{I}_{\mathrm{e}}$ coefficient A variant de 250 à 350 Par exemple, pour une masse de 15 tonnes, une réduction de la hauteur de chute de 20 à 10 mètres diminuera $\operatorname{Vr}$ de $0,5 \mathrm{~mm} / \mathrm{s}$ environ.

\section{Etude de l'influence d'une tranchée}

Les ondes de Rayleigh se propageant en surface du terrain, une tranchée de 1,50 à $2,50 \mathrm{~m}$ de profondeur permettra d'atténuer une partie de celles-ci.

La figure 4 présente les résultats obtenus sur le chantier de Plochingen.

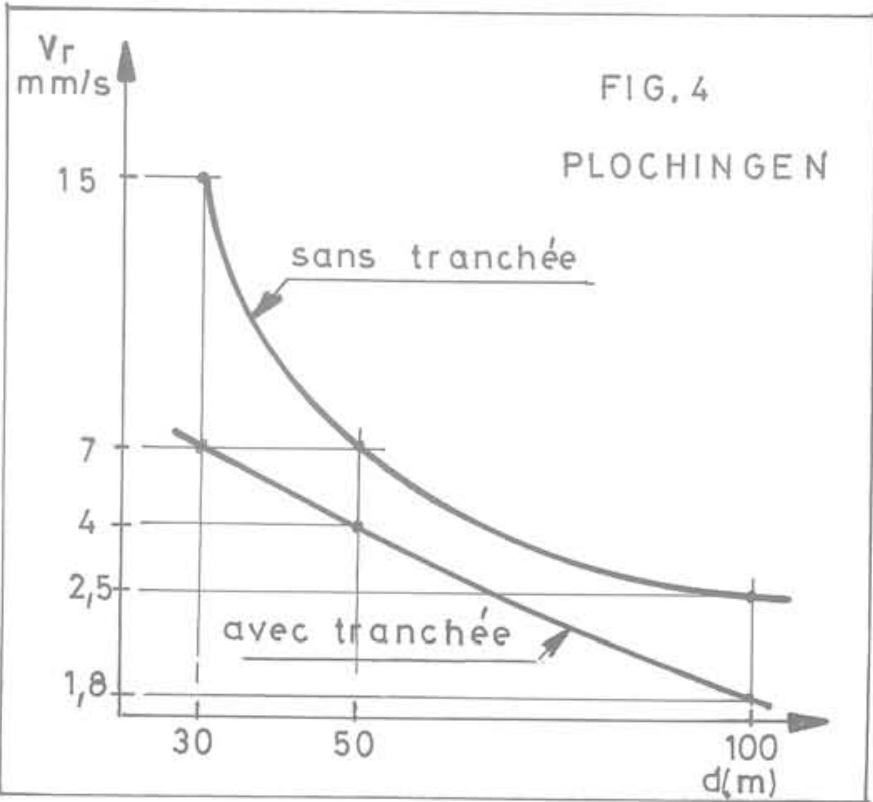

Fig. 4 - Influence d'une tranchée

La vitesse particulaire est réduite dans une proportion de 40 à $50 \%$ mais il faut préciser que cette atténuation n'est sensible qu'à proximité immédiate de la tranchée et qu'elle est susceptible de varier suivant les conditions locales.

\section{CONCLUSION}

Le résultat le plus intéressant de cette analyse est sans nul doute la courbe d'atténuation des vibrations en fonction de la distance (fig.2) - Les valeurs obtenues par l'équation sont susceptibles d'être légèrement majorées ou minorées suivant les conditions locales; cependant elles montrent que des ouvrages "normalement sensibles" situés à plus de 30 mètres du point d'impact ne doivent pas subir de dommages. $(V x<8 \mathrm{~mm} / \mathrm{s})$

Les figures 3 et 4 montrent que sans jouer sur I'éloignement, dont on n'est pas toujours mâtre, les vitesses particulières 
peuvent être réduites dans une plus petite proportion par la diminution de l'energie wnitaire et éventuellement par le creusement de tranchées anti-vibratiles.

Références

Barkan ,D.D. : Dynamics of base and foundations - New York Mac Graw Hill (1962)

Frank, A. und

Varaksin S. : Verdichtung von Böden durch dynamische Einwirkung mit Fallgawichten über und unter Wasser Bautechnik (Septembre 1977)

Landesgewerbeanstalt Bayern, Rapports de mesures de vibrations sur chantiers de consolidation dynamique (1978-79-80)

Ménard, L. La consolidation dynamique des sols de fondations - Annales ITBTP 1974 p $194-222$

Niedersächsisches Landesamt für Bodenforschung Hannover - Rapports mesures vibrations "HannoverDöhren" - Novembre 1979

Richart, F.E. Hall I.R. and Woods R.D. Vibration of soils and foundations Prenctice Hall. 